\title{
LA LEY GENERAL DE EDUCACIÓN Y LA IGLESIA: ENCUENTROS Y DESENCUENTROS
}

\author{
The General Education Act and the Church: \\ Agreements and disagreements
}

\section{Isabel Grana Gil ${ }^{\alpha}$}

Fecha de recepción: 16/12/2020 • Fecha de aceptación: 10/01/2021

Resumen. La importancia e influencia que ha tenido la Iglesia católica en la educación en España a lo largo de los siglos es de todos conocida, al igual que el hecho de que periódicamente ha habido sectores que han cuestionado su papel en la educación. El objetivo del presente artículo es conocer la postura de la Iglesia, en especial de la jerarquía eclesiástica, ante la Ley General de Educación aprobada el 4 agosto de 1970 y su posterior desarrollo. Veremos en primer lugar lo que opinaba la Iglesia ante los cambios que se avecinaban y la necesidad de estos, así como cuáles serían los puntos de inflexión. Analizaremos la dicotomía entre la enseñanza estatal y no estatal y el problema de la gratuidad de la enseñanza que se plantea, y cómo su desarrollo va a ser definitivo en el cambio de actitud de la Iglesia. Por último, haremos referencia a las distintas alternativas que se propusieron a la ley entre las que por supuesto, hablaremos de las de la enseñanza no estatal y destacaremos la de la Iglesia. Para ello, vamos a recurrir, siempre que nos sea posible, a las fuentes originales, como los informes emanados de la Comisión Episcopal de Enseñanza y Educación Religiosa que era la encargada de informar de la postura de la Iglesia ante los temas educativos, que en estos años fueron muy prolijos, y demás publicaciones de la época.

Palabras clave: Iglesia; Estado; educación; franquismo y educación; Ley General de Educación (1970).

Abstract. The importance and influence that the Catholic Church has had on Education in Spain over the centuries is well known, as is the fact that there have periodically been sectors that have questioned its role in education. The objective of this article is to examine the position of the Church,

\footnotetext{
a Departamento de Teoría e Historia de la Educación y MIDE, Facultad de Ciencias de la Educación, Universidad de Málaga, Campus de Teatinos s/n, 29071 Málaga. España. imgrana@uma.es (iD https://orcid.org/0000-0002-42398649
}

Cómo citar este artículo: Grana Gil, Isabel. «La Ley General de Educación y la Iglesia: Encuentros y desencuentros». Historia y Memoria de la Educación 14 (2021): 143-175 
especially the ecclesiastical hierarchy, with regard to the General Education Act approved on August 4, 1970 and its subsequent development. We will first look at the Church's thoughts about the changes to come and the need for them, as well as what it considered to be the turning points. We will analyze the dichotomy between state and non-state education and the issue of free education that arises, and how its development would prove definitive in the change of attitude adopted by the Church. Finally, we will refer to the different alternatives to the Law that were proposed, including those involving non-state education and education by the Church, which we will focus special attention on. To do this, we will resort, wherever possible, to original sources, such as the reports emanating from the Episcopal Commission for Teaching and Religious Education, which was in charge of reporting the Church's position on educational issues and was very active during these years as well as other publications of the time.

Keywords: Church; State; education; francoism and education; General Education Act (1970).

\section{INTRODUCCIÓN}

A lo largo de la historia ha quedado patente la mayor o menor influencia que la Iglesia católica ejercía sobre todas aquellas materias que consideraba de su competencia. La educación es uno de los campos donde esta influencia ha estado más clara, lo que ha traído tensiones por parte del Estado y la Iglesia. En general, las relaciones entre el Estado y la Iglesia han sido complejas, a menudo difíciles, pero siempre han tenido una enorme repercusión en la política educativa de las distintas épocas. ${ }^{1}$ Precisamente el primer tercio del siglo XX, hasta 1939, fue una época, no sería la primera ni la última, en la que se cuestionó el papel de la Iglesia en la educación, ya que parte de la sociedad defendía el papel protagonista del Estado como única garantía para conseguir la universalización de la educación. No obstante, desde el comienzo del franquismo, sobre todo a partir de la derrota de Hitler y Mussolini, esa importancia e influencia van a volver con mayor rotundidad debido al pacto acordado en 1953 entre la Iglesia católica y el Estado español. El segundo va a adquirir un papel subsidiario en educación, dejando de hecho la educación en manos

\footnotetext{
${ }^{1}$ Francisco Martín Zúñiga e Isabel Grana Gil, «Simposio El control del profesorado no universitario en España (1857-1931)», XX Coloquio de Historia de la Educación. Imágenes, discursos y textos en Historia de la Educación. Retos metodológicos actuales (Madrid: SEDHE, Universidad de Alcalá, 2017), 35.
} 
de las entidades privadas y sobre todo de la Iglesia, que era la institución mejor posicionada para poder hacerse cargo de esta. ${ }^{2}$

Sin embargo, décadas después, ambas posturas van a ir separándose de nuevo, sobre todo a partir del Concilio Vaticano II (1962-1965), donde va a quedar claro que ambas instituciones están en dos niveles diferentes y hasta contrapuestos. Y esta separación se va a ver claramente en el campo de la enseñanza, donde se libró la batalla más sutil entre Estado e Iglesia. Por ello, no es de extrañar que a pesar de que poco a poco esta última iba alejándose ideológicamente del franquismo y abriéndose a la sociedad, no obstante, no quisiera renunciar en ningún momento a su fuerte y privilegiada presencia en la educación. ${ }^{3}$

El objetivo del presente artículo es conocer la postura de la Iglesia, en especial de la jerarquía eclesiástica, ante la Ley General de Educación (LGE) aprobada el 4 agosto de 1970 y su posterior desarrollo. Veremos, en primer lugar, lo que opinaba la Iglesia ante los cambios que se avecinaban y la necesidad de estos, así como cuáles serían los puntos de inflexión. Analizaremos la dicotomía entre la enseñanza estatal y no estatal y el problema de la gratuidad de la enseñanza que se plantea, y cómo su desarrollo va a ser definitivo en el cambio de actitud de la Iglesia. Por último, haremos referencia a las distintas alternativas que se propusieron a la ley entre las que por supuesto, hablaremos de las de la enseñanza no estatal y destacaremos la de la Iglesia. Para ello vamos a recurrir, siempre que nos sea posible, a las fuentes originales, como por ejemplo a los informes emanados de la Comisión Episcopal de Enseñanza y Educación Religiosa que era la encargada de precisar la postura de la Iglesia en los temas educativos -en estos años muy prolijos- y demás publicaciones de la época.

\section{TENSIONES ENTRE EL ESTADO Y LA IGLESIA EN EL SEGUNDO FRANQUISMO. EL CONCILIO VATICANO II}

A menudo se presenta una imagen monolítica de la posición de la Iglesia en relación con el régimen de Franco. Por un lado, la propia Iglesia

\footnotetext{
2 Diego Sevilla Merino, «La difícil translación a una política de gobierno de un acuerdo constitucional forzado. La Unión de Centro Democrático y su malograda Ley Orgánica del Estatuto de Centros Escolares (LOECE) de 1980», Historia y Memoria de la Educación 3 (2016): 51.

${ }^{3}$ Sevilla, «La difícil translación a una política de gobierno de un acuerdo constitucional forzado», 51.
} 
trata siempre de ocultar o suavizar las diferencias y divisiones internas para dar una imagen unitaria y sólida. Por otra parte, desde fuera, especialmente desde posiciones críticas, se tiende a considerar la postura de la Iglesia como una posición única, mirando sobre todo las declaraciones oficiales y públicas de la jerarquía. Pero la verdad es que, a lo largo del franquismo y, en la historia en general, se puede observar una gran variedad de posiciones y tensiones internas entre los protagonistas. El Concilio Vaticano II será el punto de partida que haga patente y agrave las tensiones y la división entre la Iglesia y los católicos y el Estado español.

Dentro del mismo, las declaraciones Dignitatis humanae, sobre la libertad religiosa, y la constitución Gaudium et Spes sobre la relación de la Iglesia con el mundo, afectaban directamente al fundamento doctrinal y al estatus político de un régimen que se definía como católico, pero que no estaba de acuerdo con la nueva autopercepción de la Iglesia. Este desajuste entre la proclamada identidad católica de Régimen y la nueva doctrina de la Iglesia es lo que explica la tensión Iglesia-Estado en los últimos gobiernos de Franco. Además, se abre y extiende progresivamente una división, dentro de la misma Iglesia, entre proconciliares y anticonciliares, paralela a otra entre franquistas y antifranquistas, y en medio de estos polos queda un abanico de posturas. Una división algo soterrada o silenciada y, por ello, aparentemente menor.

En los últimos quince años del Régimen, los que van desde principio de la década de los sesenta hasta la muerte de Franco en 1975, se puede observar, según Feliciano Montero García ${ }^{4}$ la progresiva evolución de la tensión intraeclesial y eclesio-gubernamental en torno a unos acontecimientos a los que vamos a referirnos muy brevemente.

En primer lugar, la llamada «crisis de Acción Católica», que en realidad fue un conflicto entre la jerarquía y los seglares, que tiene lugar entre 1966 y 1968, año en el que termina el Concilio.

En el mítico «68» estudiantil se planteó en la Facultad de Teología de la Universidad Pontificia de Salamanca una huelga de estudiantes que

\footnotetext{
${ }^{4}$ Feliciano Montero García, «La Iglesia dividida. Tensiones intraeclesiales en el segundo franquismo. (La crisis postconciliar en el contexto del tardofranquismo)», en De la cruzada al desenganche: la iglesia española entre el franquismo y la transición, coords. Manuel Ortiz Heras y Damián A. González (Madrid: Sílex, 2011), 51-54.
} 
propugnaba un cambio completo de los contenidos de la enseñanza en la línea de la renovación conciliar, así como del gobierno de la universidad.

En estos mismos años crece la tensión en el interior de la Compañía de Jesús entre los partidarios de la misión obrera y del diálogo fe-secularidad y los que rechazan estos cambios.

Pero es en 1971-1972 cuando la tensión y división dentro del seno de la Iglesia llega a sus máximos en la Asamblea Conjunta del Clero y los Obispos celebrada en septiembre de 1971, donde queda patente la división de la jerarquía en relación con la aplicación pastoral del Concilio y sobre todo la legitimación o crítica del Régimen. A diferencia de los años sesenta, en ese momento la división no es tanto entre la jerarquía y los seglares de la Acción Católica, sino entre sectores del clero y de la jerarquía y, la tensión con el Régimen no se plantea solo desde los sectores renovadores del clero y los seglares, sino desde la propia jerarquía eclesiástica.

El proceso de negociación de un nuevo concordato, ${ }^{5}$ o de reforma del existente (1953), revela muy bien esa tensión creciente entre el Régimen y la jerarquía eclesiástica, a la vez que la división entre los distintos sectores del catolicismo sobre la oportunidad, la conveniencia y los términos de esa negociación. En resumen, se puede decir que la negociación del nuevo concordato obedeció sobre todo al interés del propio Gobierno, necesitado de adaptar la naturaleza católica del Régimen a la nueva situación de la Iglesia conciliar para así continuar obteniendo su apoyo y legitimidad. En la Iglesia, por su parte, se produjo una cierta división de criterios y posiciones entre los partidarios -especialmente en la Secretaría del Estado del Vaticano (monseñor Casaroli)- de aprovechar la oportunidad para establecer un nuevo estatuto de relaciones sobre la base de una mayor independencia (por ejemplo, en el nombramiento de obispos), así como de la continuidad de una protección para la presencia pública de la Iglesia, especialmente en el sistema educativo y, en definitiva, de una amigable colaboración recíproca en el horizonte incierto de un postfranquismo, y, por otra parte, la resistencia de la jerarquía

\footnotetext{
${ }_{5}^{5}$ Cfr. Pablo Martín de Santa Olalla, La Iglesia que se enfrentó a Franco. Pablo VI, la Conferencia Episcopal y el Concordato de 1953 (Madrid: Sílex. 2005); Romina Di Carli, El derecho a la libertad religiosa en la transición democrática de España (1963-1968) (Madrid: CEPC, 2009); Vicente Jesús Díaz Burillo, Las transiciones de la Iglesia (1962-1987). Del repliegue a la revancha (Granada: Comares, 2019).
} 
española que quería separarse del Régimen y del catolicismo progresista, que abogaba por una ruptura total.

Pero el Concilio y la crisis de la Dictadura no solo generarían dos Iglesias sino varias: una primera conservadora, franquista y quizá pequeña; una segunda, numerosa, puede que mayoritaria en determinados ámbitos y circunstancias, conservadora pero apolítica que, como mucho, apostaba por el Régimen porque aseguraba el statu quo; y finalmente, dos sectores renovadores, uno más moderado, reformista y comprometido socialmente, y otro radical y minoritario. De esta manera, ante la pregunta de cuál fue el comportamiento de la Iglesia - ¿continuidad o cambio? - no existe una respuesta clara ya que hay una fuerte evidencia tanto de una como del otro durante el período 1960-1975. ${ }^{6}$

Aun así, desde el punto de vista de los intereses de la jerarquía eclesiástica, la postura estaba clara y la separación institucional "amistosa» del régimen no debería desmantelar el apoyo social a la religión mayoritaria de los españoles en el terreno, por ejemplo, de la educación. Esa era parte de la contradicción en la que se movían desesperadamente los gobernantes españoles que se sentían entre perplejos y traicionados. ${ }^{7}$

\section{POSTURA DE LA IGLESIA ANTE LA LEY GENERAL DE EDUCACIÓN}

El 25 de enero de 1959, con el anuncio de la celebración de un concilio ecuménico, Juan XXIII daba el primer paso para llevar a la Iglesia católica hacia una profunda reflexión sobre sí misma y sobre la realidad histórica en la que tenía que vivir su presente.

Las declaraciones Dignitatis humanae y la Gravissimum educationis, sobre la educación cristiana de la juventud, profundizaron y desarrollaron los aspectos principales de una crisis que la industrialización había planteado a la Iglesia desde finales del siglo XIX, y en la que la vertiginosidad del progreso científico y tecnológico estaba alejando cada vez más al hombre de una visión trascendental de la vida. Entre las posibilidades de acción que el mundo proporcionaba entonces a la Iglesia, la

\footnotetext{
6 Manuel Ortiz Heras, «La Iglesia, ¿rompió con el franquismo?», Historia del Presente 15 (2010): 145.

${ }_{7}$ Feliciano Montero García, La Iglesia: de la colaboración a la disidencia (1956-1975), la oposición durante el franquismo (Madrid: Ediciones Encuentro, 2009). Recuperado de https://elibro--net.uma. debiblio.com/es/ereader/uma/61650?page=273 (consultado el 10-11-2020).
} 
enseñanza pareció ser la más apta para preservar a las jóvenes generaciones -y, por lo tanto, a la sociedad del futuro- en su arraigo en la tradición y en los valores cristianos. ${ }^{8}$

Los derechos, a la educación en general y a la educación cristiana en particular, fueron presentados como derechos inalienables del hombre en cuanto persona y miembro de la comunidad eclesial. Derechos, además, cuya diferente finalidad -respectivamente, la de formar «la persona humana en orden a su fin último y al bien de las sociedades» ${ }^{9}$ y el de contribuir «al crecimiento del Cuerpo místico [...] y [...] a la conformación cristiana del mundo»-10 introducían una importante distinción entre las competencias escolares del Estado y las de la Iglesia. Si al primero pertenecía proporcionar todos los medios para que el derecho de todos a la educación no fuese meramente nominal, a la segunda pertenecía infundir, en cualquier proceso educativo, una visión de la vida capaz de llevar al hombre a operar la necesaria síntesis cultural entre lo temporal y lo espiritual. La escuela, por lo tanto, revestía una función especial, ya que la Iglesia podía actuar en ella desde dos perspectivas diferentes: la de la enseñanza moral y religiosa en todo centro docente, por un lado, y la de una formación cristiana más completa en los centros docentes confesionales, por otro. ${ }^{11}$

Como sostiene De Carli, ${ }^{12}$ no cabe duda de que las repercusiones del Concilio Vaticano II fueron, en cierto sentido, demoledoras para las estructuras sociopolíticas de un Régimen que, como el franquista, empezaba a hacer evidente su anacronismo. Vano resultó el intento del Estado confesional de asimilar los preceptos conciliares para ajustar sus proyectos de modernización a los aires de renovación de la Iglesia: tras el Vaticano II, Iglesia y Estado parecían estar situados en dos niveles muy diferentes.

\footnotetext{
${ }^{8}$ Romina De Carli, «La enseñanza en las negociaciones concordatorias: del Concilio Vaticano II a los Acuerdos de 3 de enero de 1979». https://www.ucm.es/data/cont/docs/297-2013-07-29-2-04.pdf. -Archivo PDF: 2-3 (consultado el 15-11-2020).

9 «Gravissimus educationis», en Concilio Vaticano II. Constituciones, Decretos, Declaraciones (Madrid, BAC, 1965), 704.

10 «Gravissimus educationis», 706.

11 «Gravissimus educationis», 712-713.

12 De Carli, «La enseñanza en las negociaciones concordatorias», 3-4.
} 
En España, no hay duda de que fue en el campo de la enseñanza donde se libró la batalla más sutil entre Estado e Iglesia, y donde esta pudo explicar mejor el significado y alcance que, en sus relaciones con el poder temporal, iban a tener los principios de independencia y mutua colaboración de los que hablaba el Concilio.

Por otro lado, la política desarrollista llevada a cabo por los gobiernos tecnócratas de los años sesenta había contribuido a mejorar notablemente el nivel económico y las condiciones de vida de España. Sin embargo, la rapidez con la que se estaba llevando a cabo la industrialización no dejó de plantear profundos problemas estructurales al Régimen de Franco y, entre ellos, el de proporcionar a la sociedad una formación técnica y cultural capaz de satisfacer las exigencias de un sistema económico en el que la industria y el sector servicios iban a tener un papel más relevante que la agricultura. Por eso y, en vísperas de que la Sesión Plenaria de las Cortes votara el II Plan de Desarrollo, en el Consejo de Ministros, que se celebró en el Pardo el 5 de febrero de 1969, se dio amplio espacio a los temas relacionados con la enseñanza. En aquella ocasión el ministro de Educación y Ciencia, Villar Palasí, presentó un estudio sobre La educación en España -el conocido como «Libro Blanco»- para señalar las líneas principales de la futura y necesaria reforma del sistema educativo. ${ }^{13}$

El 4 de marzo de ese año, casi de forma paralela, se presentó a los medios informativos el documento La Iglesia y la Educación en la Espa$\tilde{n} a, h o y$, por parte de la Conferencia Episcopal. En este se lleva a cabo una relación de principios básicos de la postura de la Iglesia sobre la educación en España en esos momentos de cambio tanto a nivel educativo como social y económico del país. En este sentido, y después de reconocer y dejar constancia de que «hemos de confesar que nos hemos adormecido a veces en la confianza de nuestra unidad católica, amparada por las leyes y por tradiciones seculares. Los tiempos cambian», ${ }^{14}$ lo que se pretende es "ofrecer criterios y orientaciones para promover una presencia y una acción de la Iglesia y de los católicos españoles más

\footnotetext{
${ }^{13}$ La educación en España. Bases para una política educativa (Madrid: Ministerio de Educación y Ciencia, 1969).

${ }^{14}$ Colección de Encíclicas y Documentos Pontificios (Madrid: Acción Católica Española, 1967). Suplemento a la sexta edición, 15 .
} 
adaptadas a las nuevas necesidades que en el campo de la educación descubrimos»..$^{15}$ Ante la pregunta sobre la relación de este documento con el «Libro Blanco», el padre José Manuel Estepa, delegado general de la Comisión Episcopal de Enseñanza y Educación Religiosa, manifestó:

no significa este documento una repuesta al "Libro Blanco". Comenzó a ser preparado en abril de 1966. No es exhaustivo. Tampoco es una síntesis de doctrina que pretenda iluminar al "Libro Blanco", si bien puede constituir, de cara a la nueva ley de bases de la educación, una valiosa suma de ideas y reflexiones que ayuden a perfilar una nueva estructura y un nuevo estilo de la educación del pueblo español. No ha sido tenido en cuenta el "Libro Blanco de la enseñanza" para esta declaración, ya que cuando se redactaba, no se conocía.

[...]. Preocupa más el cambio de actitudes que el de métodos o nuevas técnicas educativas. En el documento episcopal no existe preocupación por lo institucional: sí porque la evolución de la educación en el país haga posible que "la Iglesia siga presente". ${ }^{16}$

Es decir, no es estrictamente una respuesta al «Libro Blanco», ya que se publica casi a la vez, pero sí es una declaración de intenciones sobre su postura ante los cambios que se avecinaban y que, en el terreno educativo, van a estar protagonizados por la LGE.

En este documento la Conferencia Episcopal va a tratar los temas que más le preocupan, y que son el resultado fundamentalmente de los debates desarrollados en el Concilio Vaticano II: la nueva situación de la sociedad española en el aspecto cultural, la nueva situación de la Iglesia, el objetivo fundamental de la educación, la educación en la fe, etc. Concretamente, en la segunda parte del documento, se va a ocupar de lo que denomina problemas especiales, y en ella va a dejar clara su postura, aunque ya lo estaba, sobre ciertos aspectos conflictivos que se trataban en el libro que acababa de publicar el Ministerio de Educación y que serviría de base para la aprobación al año siguiente de la LGE. En esta segunda parte, se va a referir a la educación en el ámbito escolar y a la

15 Comisión Episcopal de Enseñanza y Educación Religiosa, La Iglesia y la Educación en España, hoy. (Madrid: Conferencia del Episcopado Español, 1969), 13.

${ }_{16}$ Revista de Enseñanzas Medias, 202 (1969), 258-259. 
libertad religiosa y, dentro de ambos ámbitos, a la obligación de que la Iglesia procurara,

por todos los medios legítimos, que tal oportunidad (recibir una formación religiosa de acuerdo con las exigencias del bautismo) sea un hecho. Pero el aspecto más delicado de su misión no consiste en cuidar meramente de que este deber sea formalmente cumplido, sino, sobre todo, de que el contenido y el nivel pedagógico de la formación religiosa responda a las actuales necesidades de los educandos. ${ }^{17}$

Es decir, los obispos reivindican no solo que haya educación religiosa en todos los centros educativos, sino ser ellos quienes la controlen.

Respecto a la obligación del Estado, opinan que «la misión del Estado es proteger los derechos y deberes de las personas en el ámbito social y público. En el ordenamiento jurídico de una sociedad, la persona humana debe hallar respetados y protegidos eficazmente sus derechos y deberes».18 Siguiendo este argumento, dentro de estos ocupa un lugar muy importante el derecho y el deber de cada persona de practicar la religión libremente, según su conciencia y, como la vida religiosa se manifiesta de modo especial en la vida de la familia, por lo tanto, es deber del Estado garantizar legalmente que la familia pueda organizar libremente su vida y la educación religiosa de sus hijos. Ahora bien, como la educación de los hijos se realiza en gran parte en el ambiente escolar, se debe atender, por tanto, a la educación religiosa de los hijos. «La garantía del Estado ha de entenderse con todo realismo en lo que refiere a horarios, aulas, material escolar, profesorado, así como a la financiación de todos estos extremos, en igualdad de condiciones respecto a la enseñanza de las demás disciplinas». ${ }^{19}$ Además, afirman que «el Estado está obligado a ofrecer todas estas garantías y cautelas, sea cual fuere la actitud que adoptara respecto a su propia confesionalidad religiosa. Aún el Estado aconfesional está obligado a proteger los derechos de la persona

\footnotetext{
${ }_{17}$ Comisión Episcopal de Enseñanza y Educación Religiosa, La Iglesia y la Educación en España, hoy, 69.

${ }_{18}$ Comisión Episcopal de Enseñanza y Educación Religiosa, La Iglesia y la Educación en España, hoy, 70 .

${ }_{19}$ Comisión Episcopal de Enseñanza y Educación Religiosa, La Iglesia y la Educación en España, hoy, 71 .
} 
humana en materia religiosa». ${ }^{20}$ Sin embargo, vuelven a incidir en que de ninguna manera se podría entender que la enseñanza religiosa dependiera del Estado en cuanto a su contenido, orientación pedagógica fundamental, etc. De hecho, todo lo correspondiente a la educación religiosa -libros de texto, directrices metodológicas, designación de personas responsables de dicha educación, etc.- en el ambiente escolar debían depender solo y exclusivamente del Episcopado.

Pero también destacan el ambiente de libertad religiosa ${ }^{21}$ que empieza a apreciarse en el país, por lo que reconocen que, para los hijos de los padres no católicos que desean que no se dé a sus hijos una enseñanza religiosa católica, el Estado debe ofrecer legalmente la posibilidad de satisfacer el derecho de no recibir dicha enseñanza.

Otro de los capítulos lo dedica al derecho de los padres a la elección de centros docentes y a su financiación, ya que el primer e inalienable deber y derecho de estos es el de educar a sus hijos con verdadera libertad en la elección de las escuelas, ya sean estatales o no estatales. Afirma que:

en la práctica, si el Estado no financia de modo substancial la enseñanza no estatal, ésta desaparecerá como servicio a la sociedad y quedará reducida a la atención de pequeños grupos en sectores económicamente privilegiados.

Ante esta realidad, es evidente que, si en verdad se quiere salvar la libertad de elección de todos los padres, cualquiera que sea su condición socioeconómica, todos los centros, los del Estado, los privados y los de la Iglesia, han de ofrecer los servicios en similares condiciones económicas para las familias. ${ }^{22}$

\footnotetext{
${ }^{20}$ Comisión Episcopal de Enseñanza y Educación Religiosa, La Iglesia y la Educación en España, hoy, 72 .

${ }^{21}$ La Ley Orgánica del Estado de 10 de enero de 1967 reconocía la libertad religiosa en España. Cfr. José Manuel Cuenca Toribio, «Relaciones Iglesia y Estado en la España del siglo XX: 1931-1980», Hispania 40 (1980): 171.

${ }_{22}$ Comisión Episcopal de Enseñanza y Educación Religiosa, La Iglesia y la Educación en España, hoy, 94-95.
} 
En octubre de ese mismo año, se publicará el Informe de la Comisión Episcopal de Enseñanza sobre el Libro Blanco ${ }^{23}$ que se va a repetir lo ya expresado en el texto que venimos comentando, de una manera más sintética. Se añade la obligación de los centros no estatales, incluidos los de la Iglesia, de aceptar la supervisión del Estado en condiciones semejantes a las que este ejerce sobre sus propios centros, basándose en la consideración de servicio público que merece todo centro de enseñanza. ${ }^{24}$

Como podemos comprobar, se aboga por democratizar y universalizar la educación, por la igualdad de oportunidades para acabar con las injustas diferencias, por formar personas responsables y libres, pero también se enuncian perfectamente los principios irrenunciables en educación: la familia es la principal responsable de la educación de sus hijos y por lo tanto deben tener derecho a elegir el centro donde quieren que se eduquen sus descendientes. En cuanto a la financiación, reivindicará la gratuidad, no solo para la enseñanza primaria sino también para el bachillerato, la formación profesional y, por supuesto, tanto en la enseñanza estatal como en la privada y, además, van a defender la enseñanza religiosa en todo tipo de centros: privados y públicos.

$\mathrm{Al}$ año siguiente, tras finalizar los trabajos de su XII Asamblea Plenaria, la Conferencia Episcopal Española va a dar su opinión respecto a las propuestas de las Comisiones de las Cortes Españolas sobre el Proyecto de Ley General de Educación, en una nota de 11 julio de 1970, en la que «comprueban con satisfacción que se han mantenido y aun enriquecido las líneas generales que más directamente se relacionan con el concepto cristiano de la educación». ${ }^{25}$ Sin embargo, ven peligrar la financiación de los centros no estatales, en favor de los estatales lo que consideran una discriminación que atenta directamente contra la libertad de

\footnotetext{
${ }^{23}$ Comisión Episcopal de Enseñanza y Educación Religiosa, «Informe de la Comisión Episcopal de Enseñanza sobre el Libro Blanco», Ecclesia 1447 (1969): 27

${ }^{24}$ Comisión Episcopal de Enseñanza y Educación Religiosa, «Declaración de la Comisión episcopal de Enseñanza y Educación Religiosa sobre la reforma del sistema educativo (28 de octubre de 1969)», Razón y Fe n. ${ }^{\circ}$ ext. «La educación en España hoy» 884-885 (1971): 282.

${ }_{25}$ Comisión Episcopal de Enseñanza y Educación Religiosa, «Nota de la Conferencia Episcopal Española sobre el Proyecto de Ley General de Educación (julio de 1970)», Razón y Fe n. ${ }^{\circ}$ ext. «La educación en España hoy» 884-885 (1971): 283.
} 
elección de los padres, ya que los centros no estatales «quedarán obligados a mantener el sistema de una enseñanza retribuida, asequible sólo a ciertos sectores de la sociedad», ${ }^{26}$ aunque se afirma, en el último punto, que «los obispos y los educadores de instituciones de la Iglesia mantienen el propósito, [...] de atender, a pesar de todo, preferentemente a los sectores sociales más débiles». ${ }^{27}$

Se iban perfilando así las cuestiones conflictivas que Estado e Iglesia tuvieron que dirimir durante las negociaciones concordatarias de los años setenta. Pero lo que realmente estaba en juego era, según De Carli,

la posibilidad para la Iglesia de fundamentar su presencia en los sectores más desprotegidos de la pirámide social y de establecer unas relaciones más directas e influyentes con la sociedad española: si durante el auge del nacionalcatolicismo, la Iglesia y Estado se habían cogido de la mano para encauzar el progreso de la Sociedad; durante el posconcilio, la Iglesia buscaba el apoyo y la colaboración de la Sociedad para controlar, a través de las asociaciones intermedias, al Estado y evitar que éste fuera promotor de una política de corte laicista. ${ }^{28}$

En terminología actual, podríamos afirmar que la Iglesia no quería renunciar a su papel de influencer en la educación española y a lo que ello representa en la sociedad.

Finalmente, la LGE se aprueba el 4 de agosto de 1970. En su artículo 6 desarrolla dos líneas básicas para el tema que se trata en este artículo: el Estado reconoce y garantiza los derechos de la Iglesia católica en el ámbito de la educación y garantiza también la enseñanza religiosa y la acción espiritual y moral de la Iglesia en todas las instituciones de enseñanza. La ley incluía la formación religiosa en todos los niveles educativos (arts. 14, 16, 22, 30) y otorgaba a la Iglesia la competencia en la

\footnotetext{
${ }^{26}$ Comisión Episcopal de Enseñanza y Educación Religiosa, «Nota de la Conferencia Episcopal Española sobre el Proyecto de Ley General de Educación (julio de 1970)», 284.

${ }_{27}$ Comisión Episcopal de Enseñanza y Educación Religiosa, «Nota de la Conferencia Episcopal Española sobre el Proyecto de Ley General de Educación (julio de 1970)», 284.

${ }_{28}$ De Carli, «La enseñanza en las negociaciones concordatorias», 8.
} 
ordenación y supervisión de la educación religiosa prevista, así como la selección del profesorado. ${ }^{29}$

Sin embargo, a pesar de reconocer y garantizar la ley los derechos de la Iglesia, era el planteamiento económico de la reforma lo que delataba la intención del Estado de hacerse con la escolarización de los sectores más desfavorecidos de la sociedad. Percibiendo las consecuencias que esto podía implicar para la presencia de la Iglesia en una sociedad cada vez más secularizada, en la XIV Asamblea Plenaria del Episcopado, de 16 de febrero de 1971, José Manuel Estepa Llaurens, delegado general de la Comisión Episcopal de Enseñanza, y el padre Miguel Sánchez Vega, presidente de la FERE ${ }^{30}$ (Federación Española de Religiosos de la Enseñanza), presentaron una ponencia sobre «la reforma educativa española en su proceso de aplicación; significación de esta reforma y sus consecuencias para la pastoral educativa», en la que dejaban constancia de «la valoración positiva que en todo momento se hizo de la reforma propugnada y de la aceptación abierta y pública, por parte del Episcopado, de los objetivos que tal reforma perseguía», ${ }^{31}$ pero después de hacer un repaso por los distintos decretos y órdenes ministeriales, se quejan de que existe «una marcada tendencia estatificadora y de una escasa participación efectiva de las entidades educativas afectadas, sobre todo de carácter no estatal», ${ }^{32}$ y muestran su preocupación de modo especial por esa tendencia. La misma línea se seguirá en la XV Asamblea celebrada en septiembre de ese mismo año. En ella, la Comisión Episcopal de Enseñanza y Educación Religiosa, se

\footnotetext{
29 Alejandro Mayordomo Pérez, «Iglesia y Estado en la política educativa del franquismo», en Estudios sobre la secularización docente en España, coord. Javier Vergara Ciordia (Madrid: UNED, 1998): 191.

${ }^{30}$ La Federación Española de Religiosos de la Enseñanza (FERE) fue creada en 1957. El Decreto de erección por parte de la Congregación de Religiosos lleva fecha de 10 de abril. Sucede a la Federación de Amigos de la Enseñanza (FAE) creada en 1930 por el padre Pedro Poveda, fundador de la Institución Teresiana, el padre Domingo Lázaro, marianista, y el padre Enrique Herrera Oria, jesuita, para defender a los centros católicos en los años difíciles que se preveían en la II República Española. Desde 1962 la FERE pertenece a la Oficina Internacional de la Enseñanza Católica (OIEC). Cuando, en el seno de la OIEC, se crea en 1974 el Comité Europeo de la Enseñanza Católica (CEEC), la FERE se incorpora inmediatamente colaborando muy estrechamente con él en los distintos Congresos que organiza a nivel europeo cada dos o tres años.

31 Comisión Episcopal de Enseñanza y Educación Religiosa, «XIV Asamblea Plenaria del Episcopado (16 de febrero de 1971)», Razón y Fe n. ${ }^{\circ}$ ext. «La educación en España hoy» 884-885 (1971): 285.

32 Comisión Episcopal de Enseñanza y Educación Religiosa, «XIV Asamblea Plenaria del Episcopado (16 de febrero de 1971)», 285.
} 
lamentaba, después de analizar una vez más las disposiciones legales y las decisiones tomadas por distintos organismos oficiales, de que en «el horizonte de este sector de la vida de la sociedad y del catolicismo español aparecen factores desalentadores».33

Como afirma Alejandro Mayordomo, ${ }^{34}$ durante todo este proceso se observa que la Iglesia presentaba en estos años una actitud más favorable a la diferenciación de competencias educativas con el Estado, de acuerdo con una postura más crítica de su misión social y más decidida en la promoción de los derechos sociales, pero, por otra parte, procurará no perder su situación de privilegio en la enseñanza.

\section{EDUCACIÓN ESTATAL/NO ESTATAL/GRATUIDAD DE LA ENSEÑANZA}

La LGE establece en su artículo 55 que «los centros docentes podrán ser estatales y no estatales» y especifica que los primeros son los creados y sostenidos por la Administración del Estado en tanto que los no estatales, son los pertenecientes a la Iglesia, a otras instituciones o personas físicas o jurídicas, públicas o privadas.

En cuanto al cambio de denominación de enseñanza no estatal, en vez de privada, el padre Juan María Lumbreras, asesor del Sindicato Nacional de Enseñanza, que agrupaba a la escuela privada no religiosa, y colaborador de la FERE, lo justifica, aludiendo a que

entre los españoles la enseñanza privada suscita la idea de una enseñanza de «pago» [...] se debe no a la naturaleza de la enseñanza privada -preferimos por esto llamarla no estatal-sino a la discriminación injusta que aún padecen sus alumnos, sus familias, sus profesores y sus Centros, al ser excluidos de una participación justa y proporcional en el reparto de los fondos nacionales. ${ }^{35}$

\footnotetext{
${ }_{33}$ Comisión Episcopal de Enseñanza y Educación Religiosa, «Nota de la Comisión Episcopal de Enseñanza y Educación Religiosa (16 de septiembre de 1971)», 288.

${ }_{34}$ Mayordomo, «Iglesia y Estado en la política educativa del franquismo», 199-201.

35 Juan María Lumbreras Meabe, Momento actual de la enseñanza no estatal. ¿La gratuidad empresa posible? (Barcelona: FERE, 1973), 47.
} 
Asimismo, la ley establece en el artículo 94 la gratuidad de la Educación General Básica (EGB) y de la Profesional de Primer Grado en todos los centros estatales y no estatales, para lo que estos últimos serán subvencionados por el Estado. Los demás centros no estatales podrán, según el artículo 96, celebrar conciertos con el Estado relativos, entre otros aspectos, al orden económico. ${ }^{36}$ De hecho, en un primer momento los centros no estatales llegaron incluso a temer por su continuidad:

Esta es la gran incógnita. Entre la vitalidad y la aceptación social que tiene [la enseñanza privada], y que nadie puede negar, el ambiente contrario que se le ha querido crear, y una nueva Ley que estamos estrenando, uno no sabe con qué quedarse y de momento la resultante está siendo de una gran inquietud, incertidumbre, inseguridad y confusión. ${ }^{37}$

La puesta en marcha de la EGB, para conseguir la plena escolarización de la población en edad escolar obligatoria, de seis a trece años, fue uno de los objetivos fundamentales de la LGE. Sin embargo, el choque con la realidad, el creciente «déficit funcional» y las limitaciones financieras, agravadas por el «boom» demográfico de los años sesenta y la crisis económica de 1973, entre otros factores, hicieron que pronto quedara patente la imposibilidad de llevarlo a cabo por parte del Estado. Tantas dificultades trajeron consigo una política educativa de reducción de costes estatales y el consiguiente apoyo a la enseñanza no estatal, así como la relajación de las exigencias necesarias para una educación de calidad con el fin de posibilitar una enseñanza barata. ${ }^{38}$

Como afirma Antonio Viñao, a partir de 1972 se recurrió, sin ambages, a modos de escolarización más realistas como las aulas prefabricadas, utilización de aulas de preescolar o espacios destinados a otros usos como pasillos, bibliotecas, etc. y sobre todo a través de subvenciones a centros privados a los que se exigía una media de 35 a 40 alumnos/aula. Esta preferente atención a la EGB y la implantación de la gratuidad

\footnotetext{
${ }^{36}$ Ramón M. Torelló, «Enseñanza privada. Situación actual ¿Sentenciada a muerte?», Razón y Fe n. ${ }^{\circ}$ ext. «La educación en España hoy» 884-885 (1971): 232.

37 Torelló, «Enseñanza privada. Situación actual ¿Sentenciada a muerte?», 229.

38 Juan Manuel Fernández Soria, Estado y educación en la España contemporánea (Madrid: Síntesis, 2002), 143-144
} 
mediante subvenciones y ayudas a la construcción, la transformación y el funcionamiento de los centros privados produjeron dos cambios fundamentales en el proceso de escolarización. Por un lado, en la enseñanza pública se desatendió a la enseñanza preescolar para usar esos espacios para la EGB, lo que hizo que sus índices de escolarización apenas subieran en la década de los setenta.

Por otro lado, en el sector privado, la agilización de las subvenciones a la escuela privada ${ }^{39}$ explica la consolidación del sector privado, sobre todo el de Iglesia, a partir de la destitución de Villar Palasí, cuando esta adoptará una actitud más agresiva, desde el punto de vista político, que acabará dándole buenos resultados, ya que el porcentaje de escolarización en el sector privado y en estos niveles pasará del 27,8\% en 19691970 al $39,8 \%$ en $1974-1975.40$

En este sentido, su sucesor en el cargo, el ministro Cruz Martínez Esteruelas, informaba en su intervención en las Cortes el 10 de febrero de 1975 que entre las acciones prioritarias dentro del nivel de EGB y durante el ejercicio de ese año se determinaba «la intensificación de las subvenciones al sector privado en busca de la plenitud de la gratuidad. En 1975 está prevista la subvención a 34.350 unidades, lo que eleva a 1.210.000 alumnos que estudian en las mismas y supone un gasto de 8.480 millones de pesetas». ${ }^{41}$

Según la Síntesis Actualizada del III Informe FOESSA ${ }^{42}$ de 1978, la proporción del alumnado de la enseñanza no estatal se acercaba al 35\%, cuyo desarrollo habría tenido mucho que ver con el devenir de la LGE, tendiendo a aumentar su proporción en la enseñanza preescolar y primaria y en la formación profesional. En cambio, en el bachillerato,

\footnotetext{
39 Jordi Monés i Pujols-Busquets, «La Ley General de Educación de 1970. La Administración Educativa», Revista de Educación n. ${ }^{\circ}$ ext. «La Ley General de Educación veinte años después»(1992): 160.

40 Antonio Viñao Frago, «La Educación General Básica. Entre la realidad y el mito», Revista de Educación n. ${ }^{\circ}$ ext. «La Ley General de Educación veinte años después» (1992): 52-55.

${ }^{41}$ C. de P., «Breve diccionario de un período ministerial», Cuadernos de Pedagogía, 14 (1976).

42 La Fundación FOESSA (Fomento de Estudios Sociales y Sociología Aplicada) se constituyó en 1965, con el impulso de Cáritas Española, para conocer de forma objetiva la situación social de España. Son de destacar el Informe realizado en 1970 bajo la dirección de Amando de Miguel que, contrastado con el Libro Blanco, aporta una completa información de la realidad social de esta década, y el de 1978 que analiza la influencia de la LGE en la enseñanza en España durante los primeros años de su puesta en marcha.
} 
que en líneas generales no estaba subvencionado, decrecería su importancia relativa.

Sobre el alumnado asistente a este tipo de centros, la imagen, según el informe, era bastante clara: las clases sociales más altas, las que tienen mayor nivel de ingresos y estudios y los que viven en zonas urbanas más pobladas son quienes más frecuentan centros privados, especialmente de la Iglesia. Es decir, a pesar de que la enseñanza no estatal defendía la gratuidad y las subvenciones para garantizar la igualdad de oportunidades y la libertad de elección de los centros por parte de los padres y de los esfuerzos de una parte de los centros de la Iglesia por democratizar sus instalaciones para dar cabida a todo tipo de alumnado, la realidad es que este tipo de enseñanza y concretamente muchos de los centros de la Iglesia se encontraban justamente en el mismo sitio que antes de la reforma, es decir, al lado de las clases dominantes política y económicamente hablando.

En resumen, la E.P. (Enseñanza Privada) en España es una realidad palpable. Ha ejercido y ejerce un servicio al Estado. No obstante, un aspecto negativo de tal servicio es el clasismo por parte de la E.P., con el consiguiente mantenimiento y reproducción de la estructura de clases de nuestra sociedad. En este sentido, una eliminación drástica de la E.P. en aras de una socialización estatista y centralista, sin la participación de todos, carecería de sentido. Carecería de sentido una E.P. totalmente subvencionada si sigue estando al servicio de los más económicamente fuertes. ${ }^{43}$

\section{LAS ALTERNATIVAS DE LA IGLESIA A LA LEY GENERAL DE EDUCACIÓN}

En los últimos años del franquismo y primeros de la democracia, se produjeron una conjunción de factores que van a promover la renovación del panorama educativo español, entre los que destacan la difusión de las obras de autores como Freire, Freinet o Althusser, la aparición de revistas pedagógicas críticas y Las Escuelas de Verano, entre las que sobresale la asociación Rosa Sensat en Cataluña. Todo este movimiento, lo recogió el Colegio de Doctores y Licenciados de Madrid en su documento

${ }^{43}$ VV. AA. Síntesis Actualizada Del III Informe Foessa 1978 (Madrid: Fundación Foessa, 1978), 109. 
Una alternativa por la enseñanza. Bases de discusión a lo largo de 1975 y 1976 a través de su boletín. En este se partía de una descripción crítica de la situación de la enseñanza española en la que afirmaba que la LGE había fracasado en su intento de promover una democratización de la enseñanza, entroncada dentro de la propia democratización de la sociedad. Entre sus propuestas destacan la concepción de la enseñanza como servicio público, la defensa de la escuela pública, laica, única, gratuita y autogestionada, en la que solo la enseñanza pública debería recibir financiación pública, contenidos racionales y científicos, cuerpo único de enseñantes, etc. ${ }^{44}$

Dicha concepción resultaba ser un planteamiento opuesto a lo que venía siendo la educación durante el franquismo, lo que trajo fuertes reacciones en los sectores vinculados a la enseñanza privada que se veía fuertemente cuestionada. ${ }^{45}$

Simultáneamente a la Asamblea anual ordinaria del Colegio en la que se aprobó el documento que recogía todas estas ideas, un pequeño grupo de enseñantes, todos socios del Colegio, promovidos por la FERE, se reunió en el Sindicato Nacional de Enseñanza y redactó otro documento en el que denunciaba la propuesta del Colegio y ofrecía otra basada en el derecho de los padres a elegir escuelas, la necesidad de subvencionar las escuelas privadas, y el derecho a una opción ideológica de los centros. De la misma manera, la Federación Católica de Padres presentó opiniones casi idénticas a las expresadas. ${ }^{46}$

También la Iglesia va a dar su respuesta a dicha Alternativa en la «Declaración de la Comisión Permanente de la Conferencia episcopal sobre los planteamientos actuales de la enseñanza ${ }^{47}$ para luego proponer la suya propia. En su introducción establece que, aunque se tocan aspectos académicos, pedagógicos y administrativos, lo que realmente está en juego

\footnotetext{
${ }_{44}$ Ludolfo Paramio, «Implicaciones ideológicas de la Ley General de Educación», Documentación Social. Revista de Estudios Sociales y de Sociología Aplicada 23 (1976): 33-34.

${ }_{45}$ Sevilla, «La difícil translación a una política de gobierno de un acuerdo constitucional forzado», 53-54.

${ }^{46}$ Paloma O’Malley, «Testimonios críticos de la Ley. La alternativa», Revista de Educación n. ${ }^{\circ}$ ext. «La Ley General de Educación veinte años después» (1992): 333.

47 Comisión Episcopal de Enseñanza y Educación Religiosa, «Declaración de la Comisión Permanente de la Conferencia Episcopal sobre los Planteamientos Actuales de la Enseñanza», Documentación Social. Revista de Estudios Sociales y de Sociología Aplicada 23 (1976): 79-92.
} 
es la concepción misma de la educación, la figura del hombre nuevo que se quiere formar y el nuevo tipo de sociedad que se pretende construir.

Además reconoce que grupos de cristianos, algunos dedicados a la enseñanza, se plantean cómo ha de ser la presencia de la Iglesia en el mundo escolar, y cuál debe ser la orientación de las instituciones educativas cristianas, "a las que tantas veces se acusa de no haber cumplido con su misión evangelizadora».48 Asimismo, estiman que los espacios considerados específicos para la transmisión del mensaje de la fe son la familia cristiana y la comunidad parroquial, por lo que se cuestionan si se debe mantener en las actuales circunstancia la presencia de la Iglesia en el mundo escolar. $\mathrm{O}$, ante una eventual supresión de la confesionalidad del Estado, si no habría que deducir la exigencia de suprimir también la formación religiosa en el ámbito escolar. O si es todavía válida y legítima la presencia de la Iglesia en el campo educativo en forma institucionalizada a través de centros o colegios propios. Además, la Comisión reconoce que se extiende entre algunos sectores católicos un cierto sentido de culpabilidad por haber participado en décadas pasadas en actividades educativas encuadradas en sistemas escolares cuyos planteamientos y métodos se consideran en esos momentos en buena parte inaceptables, en alusión clara a la colaboración de la Iglesia con la represión llevada a cabo durante el franquismo.

Para dar respuestas a todas estas cuestiones, se alude al derecho a la educación de todos los ciudadanos

como respuesta al derecho que todos tienen a niveles básicos de formación ha de incluir el propósito efectivo de respetar otras exigencias no menos importantes, como son el derecho de todos los bautizados a una formación religiosa en el ámbito escolar, el derecho de los padres de familia a elegir el tipo de educación que deseen para sus hijos, sin discriminaciones de carácter económico, el derecho de los miembros de la sociedad o de los grupos sociales a crear centros de enseñanza al servicio de todos..$^{49}$

\footnotetext{
48 Comisión Episcopal de Enseñanza y Educación Religiosa, «Declaración de la Comisión Permanente de la Conferencia Episcopal sobre los Planteamientos Actuales de la Enseñanza», 80.

${ }_{49}$ Comisión Episcopal de Enseñanza y Educación Religiosa, «Declaración de la Comisión Permanente de la Conferencia Episcopal sobre los Planteamientos Actuales de la Enseñanza», 81.
} 
Además, se entiende que no existe la enseñanza neutra, y que siempre que se enseña se transmite, aun inconscientemente, una determinada forma de concebir la existencia humana. Por lo tanto, «el reconocimiento de la presencia de la Iglesia en el ámbito escolar se funda en el debido respeto a los derechos de unos ciudadanos concretos que son y se reconocen católicos o que aceptan la formación religiosa propuesta por la Iglesia».50

Como vemos, van a seguir defendiendo los mismos principios de los que venimos hablando a lo largo de estas páginas, pero «aparentemente proponían un acuerdo pacífico y demostraban una falta de beligerancia».51 El decano del Colegio de Licenciados de Madrid, Eloy Terrón, destacó, en una entrevista, en relación con el documento episcopal, que era muy importante y que notaba un progreso muy estimable en su postura, «si uno se pone a considerar el monopolio y la hegemonía que las organizaciones religiosas han ejercido sobre la enseñanza media en nuestro país».52

Ese mismo año, el teólogo Olegario González, va a publicar un artículo titulado "La Iglesia en la Alternativa de la Enseñanza». ${ }^{53}$ Nos parece especialmente interesante porque nos aporta el punto de vista de esos católicos, o al menos la de una parte de estos, que se replantean la posición de la Iglesia en la enseñanza dentro de la nueva sociedad que mantiene la Conferencia Episcopal, y que se van a alejar de la postura oficial de la Iglesia. ${ }^{54}$

\footnotetext{
${ }^{50}$ Comisión Episcopal de Enseñanza y Educación Religiosa, «Declaración de la Comisión Permanente de la Conferencia Episcopal sobre los Planteamientos Actuales de la Enseñanza», 86.

51 O’Malley, «Testimonios críticos de la Ley. La alternativa», 333.

52 O’Malley, «Testimonios críticos de la Ley. La alternativa», 333.

53 Olegario González de Cardedal, «La Iglesia en la alternativa de la enseñanza», Documentación Social. Revista de Estudios Sociales y de Sociología Aplicada 23 (1976): 93-118. Este trabajo fue publicado originalmente en la revista Vida Nueva en los números 1036 y 1037, de 26 de junio y 3 de julio de 1976.

${ }^{54}$ En 1976 se publicó, sin autoría, un libro titulado Enseñanza: debate público. Seminario de Educación que, junto a las diversas alternativas críticas de orientación socialista-laicista o regionalista, recogía, además de algunas de las declaraciones de la Conferencia Episcopal, y de otras instituciones y personas aquí mencionadas, varios textos alternativos de grupos católicos con planteamientos distintos a los de dicha conferencia: Comunidades Cristianas, Maestros cristianos, Religiosas de Madrid, "Otra voz de la Iglesia", HOAC, etc.
} 
Olegario González hace un análisis de la situación en ese momento y establece que existe una triple lectura, desde dentro, de la acción de la Iglesia en la educación durante los cuarenta años de la dictadura franquista, que deja clara la fractura que existe dentro de la propia institución:

a) Haber cumplido un gran servicio para con el pueblo español, transmitiéndole la fe a la vez que una formación cultural [...].

b) Haber ocupado de forma casi total el camino de la educación mediante instituciones propias de enseñanza, convirtiéndolas en una forma de poder e influencia públicas, habiendo servido en una forma directa o indirecta de apoyo moral, fundamentación teórica o legitimación ética a un régimen nacido de una guerra civil, caracterizado por formas dictatoriales, con marcado apoyo capitalista, y durante cuya permanencia han seguido en la pobreza amplios sectores de la población. [...]

c) Hay, sin embargo, otra perspectiva o clave de lectura compartida por no pocas personas de la Iglesia, [...]. Para estas personas la imagen que los medios oficiales de información han dado sobre las relaciones Iglesia-Estado sería en no pequeña parte falsa. El gobierno de Franco no habría aceptado a la Iglesia por motivos religiosos sino por motivos estrictamente tácticos. [...] A la Iglesia se la toleró porque no había más remedio, pero en el fondo, desde la convicción de que su marginación de la enseñanza hubiera sido un ideal. La enseñanza privada nacida y sostenida por los propios esfuerzos, solo al final logró tener una ayuda bajo la forma vergonzosa de las subvenciones. ${ }^{55}$

También analiza las críticas desde fuera, donde destaca que debido a la estrecha relación de la Iglesia con el régimen franquista es normal que, una vez muerto Franco y al entrar en crisis muchos de sus elementos, esa crítica salpique a la Iglesia que de hecho estuvo identificada con él. Por otro lado, también deja constancia de la existencia de la autocrítica nacida de los propios sectores de la Iglesia implicados en instituciones docentes propias que consideran que esta se ha servido en exceso de la educación como trampolín de su influencia en los grupos del poder;

\footnotetext{
55 González de Cardedal, «La Iglesia en la alternativa de la enseñanza», 7-8.
} 
que ha dejado la ciencia y el poder en las manos de los poderosos que lo tenían; que ha influenciado ideológicamente por ese cauce al país; y que, bajo la capa de libertad de elección de los padres, se han afirmado los derechos reales de los que ya los poseían sin percatarse de la marginación, incapacidad y subdesarrollo del resto. Sin embargo, matiza y recuerda que junto a «estos colegios de la Iglesia» ha existido otra serie de instituciones de esa misma Iglesia que de hecho han sido el único cauce institucional amplio y eficaz a través del cual se han integrado a la cultura el mundo rural, el mundo suburbano y el proletariado en general. También hace referencia a su presencia en la formación profesional de forma totalmente gratuita para el alumnado que accedía a ella.

Como resultado de todo lo dicho, se les plantea el interrogante: ¿se puede decir que en la España de 1976 la presencia masiva de la Iglesia en la enseñanza mediante centros propios subvencionados por el Estado sea el medio ideal para transmitir la fe, o sería más bien una posible entre muchas? Y, en el segundo caso, ¿no sería aconsejable buscar otras formas de presencia que logren los mismos fines y que no encuentren tales y tantas dificultades? ${ }^{56}$ A partir de todas estas reflexiones e interrogantes, va a proponer su Alternativa.

\section{Criterios, contenidos y cauces}

Partiendo de la base de que:

la Iglesia no puede seguir embarcada en la enseñanza privada mientras no cambien fundamentalmente los presupuestos teóricos y las condiciones concretas en las que se ha apoyado hasta ahora. Es un problema de fidelidad al Evangelio y de dignidad humana. ${ }^{57}$

Propone las siguientes seis tareas o cauces:

a) Concentración en lo que es acción evangelizadora y educadora de la fe en sentido explícito, para lo que deberá tener instituciones donde la oferta no esté condicionada por ningún otro fin que haya

\footnotetext{
${ }^{56}$ González de Cardedal, «La Iglesia en la alternativa de la enseñanza», 98-102.

57 González de Cardedal, «La Iglesia en la alternativa de la enseñanza», 106.
} 
de conseguir, centrándose en las comunidades de base, las escuelas catequéticas y de evangelización, de las Facultades de Teología, etc.

b) Distanciamiento progresivo, pero eficaz, de aquellas instituciones docentes que ha poseído como propias y, debido a su alto coste, les siga condenando a aparecer como servidoras de las clases sociales imperantes.

c) Aceptación de la gestión de otras instituciones docentes, de aquellas cuyos responsables o dueños que libremente se las confíen, para que en ellas se ofrezca una enseñanza en total conformidad con el pensamiento y la fe de la Iglesia católica. En tal caso no se es poseedor de un negocio, sino un profesional de la enseñanza. En este sentido tienen preferencia los grupos marginados, las situaciones humanas de subdesarrollo y de pobreza.

d) Crear instituciones docentes por razones de subsidiaridad con respecto a aquellos que jurídicamente podrían crearlas, pero que, de hecho, no pueden: colegios populares, escuelas de barrio, colegios rurales, enseñanza profesional, es decir, allí donde el grado de subdesarrollo no permita a los interesados el ejercicio de sus derechos.

e) Reclamación pública de una sociedad democráticamente configurada y de un Estado que no se considere «dueño y señor absoluto» de las instituciones públicas, sino más bien el gerente y garantía de los propósitos del pueblo y que anime sus iniciativas. Un Estado así constituido no podrá silenciar los grupos humanos que constituyen la sociedad, ni reclamará cumplir por sí solo la función docente, sino que integrará todas las iniciativas, ofreciendo así al país una rica variedad de proyectos humanos para que la libertad del individuo se pueda ejercer ante las posibilidades múltiples que se le ofrecen.

En este marco, el Estado debería financiar todas aquellas instituciones que cumplan la función docente, garantizando mediante los correspondientes y eficaces controles la seriedad, tanto de los métodos como de los contenidos. Él, a su vez, asumirá con su especial poder la defensa y promoción privilegiada de los sectores y grupos que en la concurrencia social tuvieran menos 
posibilidades y oportunidades para afirmarse a sí mismos. En esta perspectiva, y como un grupo más en la sociedad española, la Iglesia podría crear instituciones docentes propias. ${ }^{58}$

f) Preparación de hombres y mujeres que estén dispuestos y sean capaces de enseñar religión en los diversos marcos escolares, en un momento en el que, aceptada la libertad religiosa, será legítimo que esos marcos escolares ofrezcan la enseñanza de la religión a aquellos alumnos cuyos padres la demanden, o lo hagan ellos mismos a partir de una determinada edad.

Para conseguir que dicha alternativa no fuera únicamente un proyecto utópico, el autor es consciente de que, entre otras cuestiones no menores, sería necesario un distanciamiento con el Estado, una renuncia a instituciones rentables de poder: "esta fase de distanciamiento y demudación, por más dura y humillante que parezca, es la condición necesaria para recuperar su evangélica verdad», acompañada de «la autocrítica lúcida y sincera». .5

Más adelante la FERE presentó su propia declaración en defensa de sus centros docentes que se inicia con una reflexión sobre las características que deben tener en la nueva etapa democrática. Esa reflexión culmina en la Asamblea General de 1977 con la aprobación del documento programático «Del colegio de religiosos a la escuela de la comunidad cristiana. Nuevo rostro de la FERE» en el que se aboga por una escuela participativa, plenamente identificada como cristiana y abierta a todas las clases sociales. ${ }^{60}$

Como podemos observar, va a haber varias visiones distintas de la función de la Iglesia dentro de la educación a raíz de la LGE y la llegada de la democracia, ya que, por un lado, nos encontramos con la posición política de la Iglesia a través de la voz de sus obispos que van a seguir reclamando los privilegios que la misma ha disfrutado a lo largo de la historia más reciente y, por otro, va a haber una discusión interna, donde se van a cuestionar esos privilegios de los que han disfrutado y que

\footnotetext{
${ }^{58}$ González de Cardedal, «La Iglesia en la alternativa de la enseñanza», 108.

59 González de Cardedal, «La Iglesia en la alternativa de la enseñanza», 110-111.

${ }^{60}$ Breve historia de FERE-CECA. En: https://ecmadrid.org/es/escuelas-catolicas-de-madrid-2/historia-y-principio/breve-historia-de-fere-ceca (consultado el 5 de noviembre de 2020).
} 
están en colisión con la misión evangelizadora de la Iglesia y su opción fundamental por los más desfavorecidos.

\section{CONSTITUCIÓN DE 1978 Y ACUERDO ENTRE EL ESTADO ESPAÑOL Y LA SANTA SEDE DE 1979}

El 6 de diciembre de 1978 se aprobaba la Constitución Española, que trató de armonizar los intereses de la tradicional dualidad educativa española, escuela pública/escuela privada. En consecuencia, el artículo 27, llamado "artículo de la enseñanza», que refleja en sus enunciados las influencias políticas y religiosas de los distintos sectores que pugnaron por él, tuvo una difícil gestación debido a la negativa situación de la enseñanza y a las presiones tanto de las alternativas críticas a ella como de los sectores alineados con las posiciones de la Iglesia. Es decir, enseñanza pública/enseñanza privada con sus connotaciones: para unos de estatismo y monopolio docente y para otros de ahondamiento en las desigualdades. En dicho artículo, redactado de forma muy ambigua, se contemplan la libertad de enseñanza en su más extensa interpretación, la educación como un derecho fundamental y el papel del Estado como su garante, los fines democráticos y de solidaridad de la educación, el establecimiento de una enseñanza básica, obligatoria y gratuita, la función inspectora y homologadora de los poderes públicos, el derecho de los padres a que sus hijos reciban una formación acorde con sus convicciones religiosas y morales, la constitucionalización de la ayuda a la iniciativa privada, la autonomía universitaria, etc. ${ }^{61}$

Para la Iglesia representó un éxito ya que quedó reconocido el derecho a la libertad de enseñanza que, implícitamente, conllevaba el reconocimiento del derecho de la escuela privada, en su caso católica, a recibir la financiación estatal para el servicio prestado a la sociedad. Hay que tener en cuenta que, en los meses anteriores a la aprobación del artículo, el Episcopado había sido motor de una extensa campaña de presión llevada a cabo por un sinfín de asociaciones de padres de familia y de educadores cristianos. ${ }^{62}$ La Declaración sobre enseñanza por parte de

\footnotetext{
${ }^{61}$ Fernández Soria, Estado y educación en la España contemporánea, 145-147.

62 Di Carli, «La enseñanza en las negociaciones concordatorias: del Concilio Vaticano II a los Acuerdos de 3 de enero de 1979», 19.
} 
la Conferencia Episcopal, volvía a incidir en el concepto de la libertad de enseñanza en el que destacaba el derecho de los padres a elegir el tipo de educación y los centros que prefirieran para sus hijos, financiado por el Estado. ${ }^{63}$

Menos de un mes después de aprobarse la Constitución, el 3 de enero de 1979 y después de diez años de conversaciones, con sus más y sus menos, se firmaba el Acuerdo entre el Estado Español y la Santa Sede sobre Enseñanza y Asuntos Culturales, con el que se derogaba el concordato de 1953, aunque su entrada en vigor se aplazaría hasta pasado el mes de septiembre. En este Acuerdo se establecía, en el artículo uno, que «la educación que se imparta en los centros docentes públicos será respetuosa con los valores de la ética cristiana», así como, en otros artículos, que se incluirá la enseñanza de la religión católica en todos los centros de educación, en condiciones equiparables a las demás disciplinas fundamentales, aunque sin carácter obligatorio, pero garantizando el derecho a recibirla; que el profesorado de Religión formaría parte del Claustro de profesores de los respectivos centros; que le corresponde a la jerarquía señalar los contenidos de la enseñanza religiosa, así como proponer los libros de texto y material didáctico; y que la Iglesia puede establecer centros docentes de nivel no universitario y conserva su autonomía para establecer Universidades, Facultades, etc. para la formación de sacerdotes, religiosos y seglares. ${ }^{64}$

Es importante destacar la esencia del compromiso que el Estado asumía, ya que, independientemente de que el sistema escolar se concibiera como un sistema dual de centros de enseñanza estatales y privados concertados, el Estado se comprometía a respetar el derecho fundamental de los padres a decidir sobre la educación moral y religiosa de sus hijos. Obligación que se concretaba en el respeto de los valores de la ética cristiana por parte de la educación impartida en los centros docentes públicos, y en la inclusión de la enseñanza católica en todos los centros educativos. La Iglesia prácticamente conseguía todas sus aspiraciones.

\footnotetext{
${ }_{63}$ Comisión Episcopal de Enseñanza y Educación religiosa, «Declaración sobre la enseñanza realizada por el encargo de la Comisión Permanente de la Conferencia Episcopal Española», Boletín Oficial Arzobispado de Madrid-Alcalá 8 (1978): 546-548.

${ }^{64}$ Boletín Oficial del Estado, Instrumento de ratificación del Acuerdo entre el Estado español y la Santa Sede sobre Enseñanza y Asuntos Culturales, firmado en la Ciudad de Vaticano el 3 de enero de 1979. BOE de 15 de diciembre de 1979.
} 
Este será el marco en el que se moverán a partir de ese momento las relaciones entre el Estado y la Iglesia en cuanto a las cuestiones educativas, que no dejarán de estar acompañadas de constantes tensiones, de encuentros y desencuentros.

\section{CONCLUSIONES}

A partir de la convocatoria del Concilio Vaticano II, la Iglesia destaca la enseñanza, entre las posibilidades de acción que el mundo proporcionaba, como la más apta para formar religiosamente a las jóvenes generaciones. Este será el punto de partida que haga patente y agrave las tensiones y la división entre la Iglesia y los católicos y el Estado español.

El objetivo que tenían el Vaticano y una parte de la Jerarquía Episcopal era solidarizarse con las reivindicaciones de la sociedad española de aquel entonces y establecer unas nuevas relaciones con el Estado llena de desacuerdos y desapegos.

La publicación, por parte de la Conferencia Episcopal, del documento La Iglesia en España, hoy en 1969, deja muy clara la postura de la Iglesia ante los cambios que se avecinaban, en general y, sobre todo en educación. En posteriores escritos va a reclamar la necesidad, como ha quedado expuesto a lo largo del artículo, de democratizar y universalizar la educación y formar personas responsables y libres. Para ello, la Conferencia mantiene que la familia es la principal responsable de la educación de sus hijos y que, por lo tanto, los padres deben tener derecho a elegir el centro donde quieren que se eduquen sus descendientes, como única posibilidad de alcanzar la igualdad de oportunidades, que es uno de sus objetivos fundamentales.

En cuanto a la financiación, reivindicará la gratuidad, no solo para la enseñanza primaria sino también para el bachillerato, la formación profesional y por supuesto tanto en la enseñanza estatal como en la no estatal, y la enseñanza religiosa en todo tipo de centros: privados y públicos.

Sin embargo, la llegada de la democracia, y con ella la libertad de expresión, va a traer consigo un debate importante sobre la LGE, a la que se le acusa de haber fracasado -no podemos olvidar que es una ley 
aprobada durante la dictadura- no solo por las circunstancias políticas en las que fue aprobada, sino también por no haber cumplido con las expectativas de conseguir la igualdad de oportunidades y democratizar la educación. En este nuevo debate, se ponía sobre el tapete el tema de la enseñanza pública, que no estatal, y la enseñanza privada. Estábamos entrando en una nueva etapa política del país y la educación se convertía, una vez más, en un eje esencial para el futuro desarrollo de este.

Dentro de la postura que apoyaba la existencia tanto de la enseñanza pública como de la privada, pero financiada por el Estado para salvaguardar la libertad de elección de centros docentes por parte de los padres, se van a encontrar, entre otros grupos, la FERE y la Iglesia católica. Esta, a su vez, va a protagonizar un amplio debate interno sobre cuál debía ser su posición ante la sociedad en esta nueva etapa que comenzaba en el país a raíz de la muerte de Franco. Por un lado, como institución política que expresa su opinión a través de sus obispos va a seguir reclamando los privilegios que la misma ha disfrutado a lo largo de la historia más reciente; por otro, va a haber una parte, alentada por las conclusiones del Vaticano II, que se va a cuestionar esos privilegios de los que han disfrutado por estar en colisión con la misión evangelizadora de la Iglesia y su opción fundamental por los más desfavorecidos.

La promulgación de Constitución de 1978 y de los Acuerdos entre el Estado y la Santa Sede de 1979 serán el marco en el que se tendrán que mover, a partir de ese momento, las relaciones entre ambos en cuanto a la materia educativa. Un marco que sigue vigente actualmente. Por supuesto que el cambio de Régimen, y el carácter aconfesional del nuevo Estado constituyente en 1978, no fueron irrelevantes. No es casual que la negociación de los acuerdos avanzara rápidamente en los primeros momentos de la Transición.

Para terminar, quiero dejar constancia de que únicamente conocemos la postura oficial de la Iglesia, los documentos que publicaron, pero no conocemos las Actas de la Comisión Episcopal de Enseñanza o de la misma Conferencia Episcopal Española, ni por lo tanto el debate interno que pudiera tener lugar en ella. Por otro lado, conocemos algo, más allá de algún documento como el de González de Cardedal, sobre la postura de esa otra parte de la Iglesia menos representada por el Conferencia Episcopal y más dispuesta a romper con el franquismo y hacer 
autocrítica de su posición en la sociedad española del momento. Este análisis requiere mucho más trabajo de campo, de investigaciones que estudien la recepción del Vaticano II por parte de esa parte de la Iglesia no oficial, que sería la que plantaría cara al Régimen y a la propia jerarquía eclesiástica en todos los terrenos y más concretamente en el educativo.

A pesar de todo ello, creo que este texto podría ser útil a un público amplio, más allá de los jóvenes estudiantes tan alejados en el tiempo y desconocedores de las tensiones que existieron entre el franquismo y la Iglesia, ya que a menudo se presenta una imagen monolítica de la posición de la Iglesia en relación con el régimen de Franco. También por supuesto, como síntesis, a aquellas personas poco dedicadas al estudio de este tema.

\section{Nota sobre la autora}

Isabel Grana Gil es Doctora en Filosofía y Letras (Sección Ciencias de la Educación). Profesora Titular de Historia de la Educación en Andalucía de la Universidad de Málaga. Es directora del Departamento de Teoría e Historia de la Educación y MIDE y ha sido coordinadora del Máster en Cambio Social y Profesiones educativas de la Universidad de Málaga. Sus líneas de investigación se centran en la Depuración franquista del profesorado de enseñanza media y escuelas normales, la Iglesia y el Estado como órganos de control de la Educación y la Historia de la Educación de las mujeres. Actualmente participa investigadora principal en el proyecto titulado las ideas sobre el estado en la configuración del sistema educativo español. 1900-1931 financiado por la UMA y como investigadora del proyecto nacional Totalitarismos y exilio interior de las educadoras en España (1923-1975): silencios, resistencias y resignificaciones. Publicaciones recientes: Coordinadora del libro Investigación educativa y cambio social. Barcelona, Octaedro, 2020; ¿Qué fue de ellas? Profesoras de instituto depuradas durante el franquismo en $\mathrm{La}$ educación de las mujeres en Iberoamérica: análisis histórico. Tirant Humanidades, 2019; Una visión global de la depuración franquista del profesorado: semejanzas y peculiaridades. Revista de Estudios Regionales, 114, 2019. 


\section{REFERENCIAS}

Boletín Oficial del Estado. Instrumento de ratificación del Acuerdo entre el Estado español y la Santa Sede sobre Enseñanza y Asuntos Culturales, firmado en la Ciudad de Vaticano el 3 de enero de 1979. BOE de 15 de diciembre de 1979.

C. de P. «Breve diccionario de un período ministerial». Cuadernos de Pedagogía 14 (1976).

Colección de Encíclicas y Documentos Pontificios. Madrid: Acción Católica Española, 1967. Suplemento a la sexta edición.

Comisión Episcopal de Enseñanza y Educación Religiosa. La Iglesia y la Educación en España, Hoy. Madrid: Conferencia del Episcopado Español, 1969.

Comisión Episcopal de Enseñanza y Educación Religiosa. «Informe de la Comisión Episcopal de Enseñanza sobre el Libro Blanco». Ecclesía 1447 (1969): 27.

Comisión Episcopal de Enseñanza y Educación Religiosa. «La Iglesia y la Educación en España hoy. Declaración de la Comisión Episcopal de Enseñanza y Educación Religiosa». Revista de Enseñanzas Medias 202 (1969): 256-260.

Comisión Episcopal de Enseñanza y Educación Religiosa. «Declaración de la Comisión episcopal de Enseñanza y Educación Religiosa sobre la reforma del sistema educativo (28 de octubre de 1969)». Razón y Fe n. ${ }^{\circ}$ ext. "La educación en España hoy», 884-885 (1971): 279-283.

Comisión Episcopal de Enseñanza y Educación Religiosa. «Nota de la Conferencia Episcopal Española sobre el Proyecto de Ley General de Educación (julio de 1970)». Razón y Fe n. ${ }^{\circ}$ ext. «La educación en España hoy», 884-885 (1971): 283-284.

Comisión Episcopal de Enseñanza y Educación Religiosa. «XIV Asamblea Plenaria del Episcopado (16 de febrero de 1971)». Razón y Fe n. ${ }^{\circ}$ ext. "La educación en España hoy», 884-885 (1971): 285.

Comisión Episcopal de Enseñanza y Educación Religiosa. «Nota de la Comisión Episcopal de Enseñanza y Educación Religiosa (16 de septiembre de 1971)». Razón y Fe n. ${ }^{\circ}$ ext. "La educación en España hoy», 884-885 (1971): 287-288.

Comisión Episcopal de Enseñanza y Educación Religiosa. «Declaración de la Comisión Permanente de la Conferencia Episcopal sobre los Planteamientos Actuales de la Enseñanza». Documentación Social. Revista de Estudios Sociales y de Sociología Aplicada 23 (1976): 79-92.

Comisión Episcopal de Enseñanza y Educación Religiosa. «Declaración sobre la enseñanza realizada por el encargo de la Comisión Permanente de la Conferencia Episcopal Española». Boletín Oficial Arzobispado de Madrid-Alcalá 8 (1978): 546-548.

Conferencia Episcopal Española y Educación Religiosa. «Nota de la Conferencia Episcopal Española sobre el Proyecto de Ley General de Educación 
(julio de 1970)». Razón y Fe n. ${ }^{\circ}$ ext. «La educación en España hoy», 884-885 (1971): 283-284.

Cuenca Toribio, José Manuel. "Relaciones Iglesia y Estado en la España del siglo XX: 1931-1980». Hispania 40 (1980): 153-176.

Di Carli, Romina. "La enseñanza en las negociaciones concordatorias: del Concilio Vaticano II a los Acuerdos de 3 de enero de 1979», https:/www.ucm.es/ data/cont/docs/297-2013-07-29-2-04.pdf. Archivo PDF.

Di Carli, Romina. El derecho a la libertad religiosa en la transición democrática de España (1963-1968). Madrid: CEPC, 2009.

Díaz Burillo, Vicente Jesús. Las transiciones de la Iglesia (1962-1987). Del repliegue a la revancha. Granada: Comares, 2019.

Enseñanza: debate público. Seminario de Educación. Madrid: Acción Católica Española, 1976.

Fernández Soria, Juan Manuel. Estado y educación en la España contemporánea. Madrid: Síntesis, 2002.

González de Cardedal, Olegario. «La Iglesia en la alternativa de la enseñanza». Documentación Social. Revista de Estudios Sociales y de Sociología Aplicada 23 (1976): 93-118.

"Gravissimus educationis», en Concilio Vaticano II. Constituciones, Decretos, Declaraciones. Madrid: BAC, 1965.

La educación en España. Bases para una política educativa. Madrid: Ministerio de Educación y Ciencia, 1969.

Lumbreras Meabe, Juan María. Momento actual de la enseñanza no estatal. ¿La gratuidad empresa posible? Barcelona: FERE, 1973.

Martín Zúñiga, Francisco e Isabel Grana Gil. «Simposio El control del profesorado no universitario en España (1857-1931)», XX Coloquio de Historia de la Educación. Imágenes, discursos y textos en Historia de la Educación. Retos metodológicos actuales. Madrid: SEDHE, Universidad de Alcalá, 2017: 35-37.

Mayordomo Pérez, Alejandro. «Iglesia y Estado en la política educativa del franquismo». En Estudios sobre la secularización docente en España, coordinado por Javier Vergara Ciordia, 183-203. Madrid: UNED, 1998.

Monés i Pujols-Busquets, Jordi. «La Ley General de Educación de 1970. La Administración Educativa». Revista de Educación n. ${ }^{\circ}$ ext. «La Ley General de Educación veinte años después»(1992): 131-192.

Martín de Santa Olalla, Pablo. La Iglesia que se enfrentó a Franco. Pablo VI, la Conferencia Episcopal y el Concordato de 1953. Madrid: Sílex, 2005.

Montero García, Feliciano. «La Iglesia dividida. Tensiones intraeclesiales en el segundo franquismo. (La crisis postconciliar en el contexto del tardofranquismo)». En De la cruzada al desenganche: la iglesia española entre el franquismo y la transición, coordinado por Manuel Ortiz Heras y Damián A. González. Madrid: Sílex, 2011, 51-75. 
O’Malley, Paloma. «Testimonios críticos de la Ley. La alternativa». Revista de Educación n. ${ }^{\circ}$ ext. "La Ley General de Educación veinte años después» (1992): 325-337.

Ortiz Heras, Manuel. «La Iglesia, ¿Rompió con el franquismo?». Historia del Presente 15 (2010): 143-152.

Paramio, Ludolfo. «Implicaciones ideológicas de la Ley General de Educación». Documentación Social. Revista de Estudios Sociales y de Sociología Aplicada 23 (1976): 25-36.

Sevilla Merino, Diego. «La difícil translación a una política de gobierno de un acuerdo constitucional forzado. La Unión de Centro Democrático y su malograda Ley Orgánica del Estatuto de Centros Escolares (LOECE) de 1980». Historia y Memoria de la Educación 3 (2016): 45-70.

Torelló, Ramón M. «Enseñanza privada. Situación actual ¿Sentenciada a muerte?». Razón y Fe 884-885 (1971): 209-235.

Viñao Frago, Antonio. «La Educación General Básica. Entre la realidad y el mito». Revista de Educación n. ${ }^{\circ}$ ext. "La Ley General de Educación veinte años después» (1992): 47-72.

VV. AA. Síntesis Actualizada Del III Informe Foessa 1978. Madrid: Fundación Foessa, 1978. 